\title{
Planetary Gearbox Vibration Signal Characteristics Analysis and Fault Diagnosis
}

\author{
Qiang Miao ${ }^{1,2}$ and Qinghua Zhou ${ }^{2}$ \\ ${ }^{1}$ State Key Laboratory of Mechanical Transmission, Chongqing University, Chongqing 400044, China \\ ${ }^{2}$ School of Aeronautics and Astronautics, Sichuan University, Chengdu, Sichuan 610065, China \\ Correspondence should be addressed to Qiang Miao; mqiang@scu.edu.cn
}

Received 26 June 2015; Accepted 20 August 2015

Academic Editor: Chuan Li

Copyright ( 2015 Q. Miao and Q. Zhou. This is an open access article distributed under the Creative Commons Attribution License, which permits unrestricted use, distribution, and reproduction in any medium, provided the original work is properly cited.

Planetary gearboxes are widely used in helicopters, wind turbines, mining machinery, and so forth. The structure and motion type of a planetary gearbox are more complex in comparison with a fixed-shaft one, which makes condition monitoring and fault diagnosis of planetary gearbox a challenging issue in practical applications. In order to understand the fundamental nature of planetary gearbox vibration, this paper conducts an investigation on vibration characteristics of a single-stage planetary gearbox. Assuming that the gearbox and the sensor revolve inversely at the speed of planet carrier, the problem can be transformed into two easier parts: research on fixed-shaft gearbox signal model and research on influence of sensor spinning. Based on this assumption, a vibration signal model of planetary gearbox is obtained. Experimental data are used to validate the model.

\section{Introduction}

The development of equipment manufacturing industry raises urgent demand on high performance transmissions. Compared with traditional fixed-shaft gearing systems, a planetary gearbox has compact structure and light weight, while its load-carrying capacity, transmission precision, and efficiency are much higher. Therefore, planetary gearboxes have been widely used in helicopters, wind turbines, mining machinery, and so forth. However, the reliability of these high performance transmissions is a critical issue in practical applications, because the occurrence of gearbox failure may lead to catastrophic accidents and cause severe economic losses. Therefore, it is necessary to conduct planetary gearbox condition monitoring and fault diagnosis so as to reduce equipment operational cost and risk.

In general, a planetary gear train in a gearbox consists of a sun gear, a ring gear, a planet carrier, and several planet gears. Compared with a fixed-shaft gearing system, a planetary gear train has a composite rotating motion style. Planet gears revolve on their own shafts and along central sun gear. The composite motion caused by multi-gear-meshing results in time-variant vibration propagation paths, and this generates distinctive nonstationarity in planetary gearbox dynamic response. The composite motion induced nonstationarity is coupled with nonstationarity caused by gear train failure, which makes it a challenging problem in planetary gearbox condition monitoring.

Gearbox dynamic response analysis is an essential step to reveal fault root causes and their response features. For example, Ericson and Parker [1] investigated the impact of torque changes on planetary gear natural frequencies, mode shapes, and damping parameters. Bartelmus et al. [2] conducted fault detection and diagnosis on fixed-shaft and planetary gearboxes under time-varying nonstationary operations and established gearbox dynamics models to understand the fault related phenomena through vibration signal analysis. Gu and Velex [3] proposed a lumped parameter model to analyze the influence of planet position errors in planet gears. It should be noted that a gearbox dynamics model should be built on the basis of certain simplifications, weakening its feasibility under very complicated working environments.

In planetary gearbox condition monitoring and diagnosis, an early research is to separate meshing vibration signals of planet gear and sun gear through the time-domain averaging (TSA) technique [4]. Based on this idea, Forrester 
[5] proposed a signal filtering technique to realize the timedomain averaging for each individual planet gear. However, the TSA technique is established on the basis of constant rotating speed, while speed variation in practical applications limits signal separation accuracy. Recently, a lot of research has been conducted on planetary gearbox fault diagnosis. Orchard and Vachtsevanos [6] proposed a particle-filtering based framework for helicopter planetary gear train diagnosis and prognosis. Barszcz and Randall [7] presented a tooth crack detection method in wind turbine gearboxes using the spectral kurtosis. Lei et al. [8] proposed two diagnostic parameters based on the analysis of vibration features of planetary gearboxes. Feng and Zuo [9] investigated vibration signal models for planetary gearbox fault diagnosis. Reference [10] conducts a comprehensive survey on the state-ofthe-art techniques in planetary gearbox fault diagnosis.

Engineering practices showed that vibration signal generated by different fault types and meshing components may demonstrate their respective waveform characteristics, and dynamic signal collected from equipment is an overall description of these vibration sources [11-14]. Due to the fact that a planetary gear train has complicated motion style, vibration induced by a planetary gear train differs a lot from a fixed-shaft gearing system. In order to understand the fundamental nature of planetary gear train vibration and develop reliable fault diagnosis methods, it is necessary to analyze vibration characteristics of planetary gearbox under different conditions.

In general, most of work on planetary gearbox fault diagnosis focuses on the fault feature extraction and fault recognition. The purpose of this paper is to investigate vibration characteristics of a planetary gear train, and a planetary gearing signal model was obtained by assuming that the planetary gearbox and the sensor revolve inversely at the speed of carrier. The results obtained in this research may provide a new way to understand the fault behavior and develop new fault diagnosis methods. Experimental data collected from a planetary gearbox test rig were used to validate the developed signal model in gearbox fault identification.

The rest of the paper is organized as follows. Section 2 briefly describes the assumptions that are made in this research. Section 3 gives theoretical calculation formula for planetary gear train meshing frequency and characteristic frequencies. Section 4 shows the development of vibration signal model based on the assumptions defined in Section 2. In Section 5, the planetary gearbox test rig is briefly introduced, and the vibration data collected from this test rig are used for model validation. Finally, conclusions are summarized in Section 6.

\section{Assumptions in Planetary Gearbox Vibration Characteristics Analysis}

In the process of gearbox running, the number of meshing teeth varies alternatively along with gear train rotation, which generates gear meshing vibration induced by periodic changes of tooth meshing stiffness. If a tooth fault exists in a planetary gearbox, it introduces periodic impulses or serious modulating phenomena in vibration signal, and the

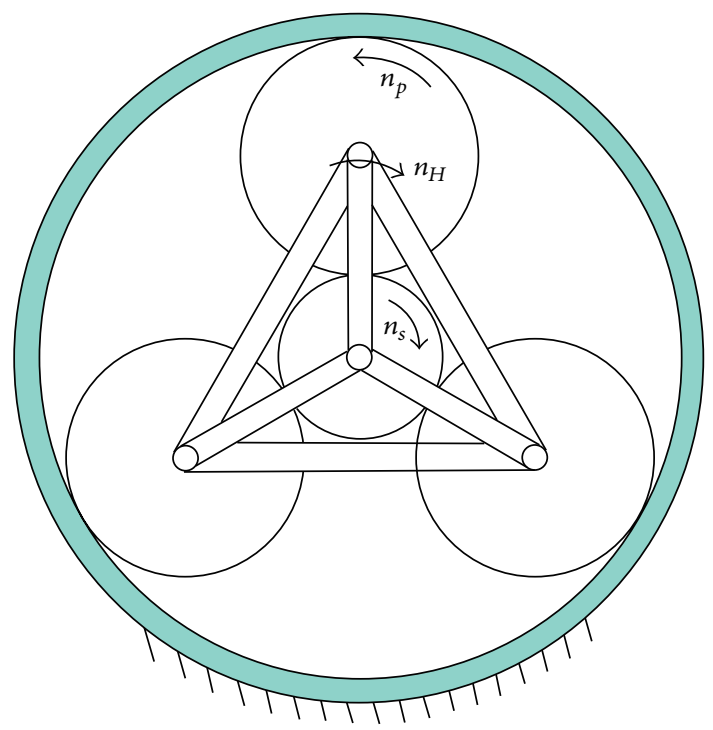

FIGURE 1: A schematic graph of planetary gear train.

corresponding characteristic frequency is associated with the rotating frequency of faulty gear relative to planet carrier [9].

Given a planetary gear train, usually the ring gear is fixed, while the carrier and the planet gears are bonded together, as shown in Figure 1. In this paper, $r, p, s$, and $H$ represent ring gear, planet gear, sun gear, and planet carrier, respectively. In addition, $N$ is the rotation speed, $z$ is the number of gear teeth, and $K$ is the number of planet gears.

In the computation of planet gear train transmission ratio, it is usually assumed that the whole gearset reversely rotates at the carrier speed, and the calculation of a planet gear train transmission ratio is transformed to a fixed-shaft gearing one. Similarly, in the analysis of planetary gearing vibration characteristics, it is assumed that the gearbox and the sensor rotate reversely with the speed of planet carrier. Then, the analysis of planetary gearbox vibration can be divided into two simple parts: the fixed-shaft gearset vibration analysis and the impact of sensor's spinning motion on fixed-shaft gearset vibration signal. Unless otherwise specified, the term "relative" in this paper means the relative motion to planet carrier.

\section{Planetary Gear Train Meshing Frequency and Characteristic Frequencies}

3.1. Gear Meshing Frequency. When a planet gear completes one relative revolution, it has the same number of meshing teeth with the sun gear and the ring gear. Therefore, the meshing frequency of sun gear to planet gear is equal to the meshing frequency of planet gear to ring gear. Given the planetary gear train shown in Figure 1, if the rotation frequency of sun gear's shaft is known as $f_{s}$, then the meshing frequency $f_{m}$ can be calculated as

$$
f_{m}=f_{H} \cdot z_{r}=\frac{z_{r} z_{s}}{z_{r}+z_{s}} \cdot f_{s} .
$$




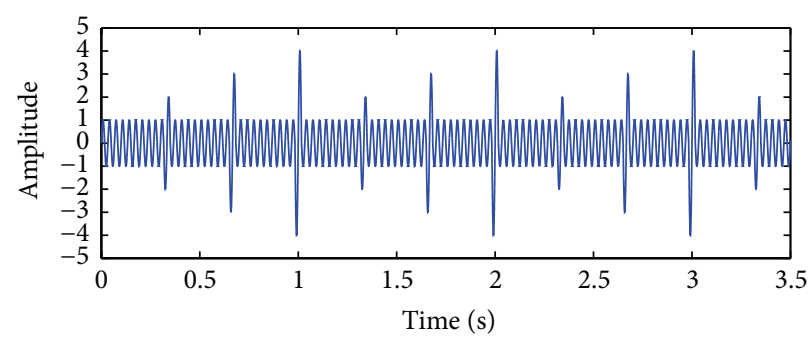

(a)

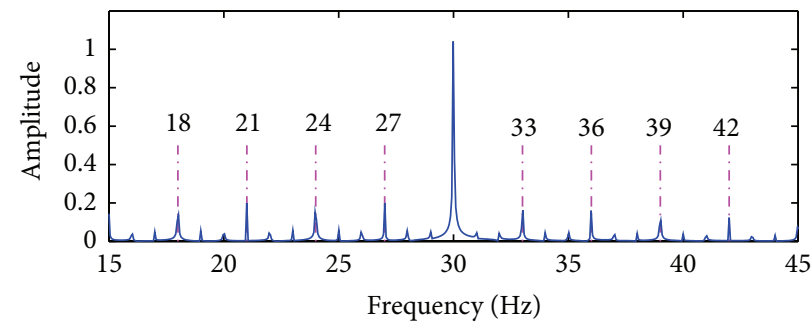

(b)

Figure 2: A simulation signal. (a) Time-domain waveform; (b) frequency spectrum.

\subsection{Characteristic Frequency of Sun Gear}

(1) Characteristic Frequency of Sun Gear with Distributed Fault. Given a distributed fault on a sun gear, the change of meshing condition is in one period when the sun gear completes one relative revolution. Then, the corresponding characteristic frequency of sun gear with distributed fault $\left(f_{\text {csd }}\right)$ is equal to the relative rotating frequency $f_{s}^{H}$ :

$$
f_{\text {csd }}=f_{s}^{H}=\frac{f_{m}}{z_{s}}=\frac{z_{r}}{z_{r}+z_{s}} \cdot f_{s} .
$$

(2) Characteristic Frequency of Sun Gear with Local Fault. Assume that a local fault exists on a sun gear. When the local fault meshes with a planet gear, the vibration signal is modulated by an impulse signal. When the local fault exits the meshing area, the vibration signal returns to its normal condition. In one revolution of the sun gear, such modulating phenomenon happens $K$ times. Assume that these modulation conditions are identical; the characteristic frequency of sun gear with local fault $\left(f_{\text {csl }}\right)$ is $K$ times of the relative rotating frequency $f_{s}^{H}$ :

$$
f_{\mathrm{csl}}=K \cdot f_{s}^{H}=K \cdot \frac{f_{m}}{z_{s}}=K \cdot \frac{z_{r}}{z_{r}+z_{s}} \cdot f_{s} .
$$

In fact, due to the existence of manufacturing and assembly errors in planet gear, planet carrier, and ring gear, the modulating phenomena in vibration signal caused by sun gear fault area meshing may not be identical. This paper utilizes a simulation signal to further discuss this case.

Take the planetary gear train shown in Figure 1 as an example. Assume that the amplitude of meshing vibration signal produced by sun gear and planet gear is 1 , the meshing frequency is $30 \mathrm{~Hz}$, and the relative rotating frequency of sun gear is $1 \mathrm{~Hz}$. When the sun gear has a local fault, the fault area meshes with 3 planet gears, and the amplitudes of resulting amplitude-modulation (AM) phenomenon produced by these meshing processes are 2, 3, and 4, respectively. For simplification purpose, the frequency modulation (FM) phenomenon is not considered, and it is assumed that the waveform of modulating signal is a square wave. Figure 2 shows the time-domain waveform and its frequency spectrum. It can be observed that the values of spectral lines at $f_{m} \pm 3 f_{s}^{H} \cdot i(i \in Z)$ are obviously large, while the other spectral lines are relatively small. Therefore, even if the manufacturing and assembly errors from planet gear, planet carrier, and ring gear are considered, the characteristic frequency of sun gear with local fault can still be treated as $K \cdot f_{s}^{H}$, which is consistent with (3).

\subsection{Characteristic Frequency of Planet Gear}

(1) Characteristic Frequency of Planet Gear with Distributed Fault. Given a distributed fault on a planet gear, the change of meshing condition is in one period when the planet gear completes one relative revolution. Thus, the corresponding characteristic frequency of planet gear with distributed fault $\left(f_{\text {cpd }}\right)$ is equal to the relative rotating frequency of planet gear $f_{p}^{H}$ :

$$
f_{\text {cpd }}=f_{p}^{H}=\frac{f_{m}}{z_{p}}=\frac{z_{r} \cdot z_{s}}{z_{p}\left(z_{r}+z_{s}\right)} \cdot f_{s} .
$$

(2) Characteristic Frequency of Planet Gear with Local Fault. Assume that a local fault exists on a single side of a planet gear tooth. When the local fault meshes with the sun gear or the ring gear, the vibration signal is modulated by an impulse signal. When the local fault exits the meshing area, the vibration signal returns to its normal condition. In one revolution of planet gear, such modulating situation happens once. Thus, the characteristic frequency of planet gear with single-sided local fault $\left(f_{\mathrm{cpl}}\right)$ is equal to the relative rotating frequency $f_{p}^{H}$ :

$$
f_{\mathrm{cpl}}=f_{p}^{H}=\frac{f_{m}}{z_{p}}=\frac{z_{r} \cdot z_{s}}{z_{p}\left(z_{r}+z_{s}\right)} \cdot f_{s} .
$$

If a local fault happens on both sides of a planet gear tooth, the vibration signal modulation happens twice during its one period revolution. Although the amplitudes of these two modulations may be different, similar results can be obtained from the discussion of sun gear local fault characteristic frequency. Therefore, the characteristic frequency of planet gear with two-sided local fault is twice the relative rotating frequency $f_{p}^{H}$ :

$$
f_{\mathrm{cpl}}=2 f_{p}^{H}=2 \cdot \frac{f_{m}}{z_{p}}=2 \cdot \frac{z_{r} \cdot z_{s}}{z_{p}\left(z_{r}+z_{s}\right)} \cdot f_{s} .
$$


3.4. Characteristic Frequency of Ring Gear. Similar to the results from sun gear characteristic frequencies, the characteristic frequency of ring gear with distributed fault $\left(f_{\text {crd }}\right)$ and the characteristic frequency of ring gear with local fault $\left(f_{\mathrm{crl}}\right)$ can be obtained, respectively:

$$
\begin{aligned}
& f_{\text {crd }}=\frac{z_{s}}{z_{r}+z_{s}} \cdot f_{s}=f_{H}, \\
& f_{\text {crl }}=K \cdot \frac{z_{s}}{z_{r}+z_{s}} \cdot f_{s}=K \cdot f_{H} .
\end{aligned}
$$

\section{Planetary Gear Train Vibration Signal Model}

4.1. Vibration Signal Model after Transforming to Fixed-Shaft Gearing System. For a pair of gears under working condition, the number of meshing teeth changes alternatively during the meshing process. This results in the periodic change of tooth meshing stiffness and causes gear meshing vibration. Due to the unavoidable errors in manufacturing and assembly process, as well as faults on gears, the gear meshing condition changes frequently. The change is closely associated with the gear relative rotating period, and it generates amplitude modulating and frequency modulating phenomena in the original signal. Thus, the vibration signal caused by faulty gear meshing can be described by amplitude and frequency modulations:

$$
x_{o}(t)=\sum_{k=0}^{\infty} a_{k}(t) \cos \left[2 \pi k f_{m} t+b_{k}(t)+\theta_{k}\right] .
$$

Here, $a_{k}(t)$ and $b_{k}(t)$ are the amplitude modulation and the frequency modulation functions, respectively:

$$
\begin{aligned}
& a_{k}(t)=A_{0}+\sum_{n=1}^{\infty} A_{k n} \cos \left(2 \pi n f_{c} t+\varphi_{k n}\right), \\
& b_{k}(t)=\sum_{l=1}^{\infty} B_{k l} \sin \left(2 \pi l f_{c} t+\phi_{k l}\right) .
\end{aligned}
$$

Here, $f_{c}$ is the characteristic frequency. $A_{0}, A_{k n}$, and $B_{k l}$ are constants, representing the amplitudes of vibration signal, $\mathrm{AM}$, and FM, respectively.

For the vibration signal collected under gear local fault, its modulating signal is a periodic impulse. Since the periodic function can be approximately expressed by an infinite series of sine functions and cosine functions, the amplitude modulating function and the frequency modulating function caused by local fault can be expressed by (10) and (11).

For simplification purpose, we only analyze the fundamental frequencies in (9) (11). That is, only the case of $k=$ $n=l=1$ is considered. Then, (9) can be simplified as

$$
\begin{aligned}
x_{o}(t)= & A_{1}\left[1+A \cos \left(2 \pi f_{c} t+\varphi_{1}\right)\right] \\
& \cdot \cos \left[2 \pi f_{m} t+B \sin \left(2 \pi f_{c} t+\phi_{1}\right)+\theta_{1}\right] .
\end{aligned}
$$

In addition, we have

$$
e^{j z \sin \varphi}=\sum_{k=-\infty}^{+\infty} J_{k}(z) e^{j k \varphi},
$$

where $J_{k}(z)$ is the $k$ th-order first kind Bessel function of the variable $z$.

According to (13), (12) can be expanded as

$$
\begin{aligned}
x_{o}(t) & \\
= & A_{1}\left[1+A \cos \left(2 \pi f_{c} t+\varphi_{1}\right)\right] \\
& \cdot \sum_{k=-\infty}^{+\infty} J_{k}(B) \cos \left[2 \pi\left(f_{m}+k f_{c}\right) t+k \phi_{1}+\theta_{1}\right] .
\end{aligned}
$$
have

Following the properties of trigonometric functions, we

$$
\cos \alpha \cdot \cos \beta=\frac{1}{2}[\cos (\alpha+\beta)+\cos (\alpha-\beta)] .
$$

Then, (14) can further be expanded as

$$
\begin{gathered}
x_{o}(t)=\sum_{k=-\infty}^{+\infty} A_{1} \cdot J_{k}(B)\left\{\cos \left[2 \pi\left(f_{m}+k f_{c}\right) t+\varphi_{k}\right]\right. \\
+\frac{A}{2} \cos \left[2 \pi\left(f_{m}+k f_{c}+f_{c}\right) t+\phi_{k}\right] \\
\left.+\frac{A}{2} \cos \left[2 \pi\left(f_{m}+k f_{c}-f_{c}\right) t+\theta_{k}\right]\right\},
\end{gathered}
$$

where $f_{c}$ is the characteristic frequency, $\varphi_{k}=k \phi_{1}+\theta_{1}, \phi_{k}=$ $k \phi_{1}+\varphi_{1}+\theta_{1}$, and $\theta_{k}=-k \phi_{1}+\varphi_{1}-\theta_{1}$. Since $A_{1}, J_{k}(B)$, and $A$ are constants, (16) can be further simplified by properties of trigonometric functions, and it can be obtained that

$$
x_{o}(t)=\sum_{k=-\infty}^{+\infty} C_{k} \cos \left[2 \pi\left(f_{m}+k f_{c}\right) t+\alpha_{k}\right] .
$$

Here, $C_{k}$ and $\alpha_{k}$ are constants that are related to $A_{1}, A,\left\{J_{i}(B)\right\}$, $\left\{\varphi_{i}\right\},\left\{\phi_{i}\right\}$, and $\left\{\theta_{i}\right\}$.

According to (17), a series of sidebands emerge around the meshing frequency $\left(f_{m}\right)$ of a faulty gear, and the interval between the neighbouring sidebands is equal to the characteristic frequency of faulty gear $\left(f_{c}\right)$. If we take into account the harmonics $n f_{m}\left(n \in N^{*}\right)$ of the meshing frequency, the sidebands emerge around the positions $n f_{m} \pm k f_{c}\left(k \in N^{*}\right)$.

4.2. Influence of Sensor Spinning to Vibration Signal. Given different vibration propagation paths, the impact of sensor spinning may not be the same. Assume that a fault exists on a sun gear. As shown in Figure 3, the vibration caused by the meshing process between sun gear and planet gear may be transmitted to the sensor through 3 paths.

In path 1 , the vibration starts from the meshing point, passes through the planet gear, the ring gear, and the case of gearbox, and finally reaches the signal measurement point. In path 2, the vibration starts from the meshing point, passes through the planet gear with its bearing, the planet carrier with its shaft, the bearing, and the case of gearbox, and finally reaches the signal measurement point. In path 3 , the vibration starts from the meshing point, passes through the sun gear, the shaft of sun gear with its bearing, and the case of gearbox, 


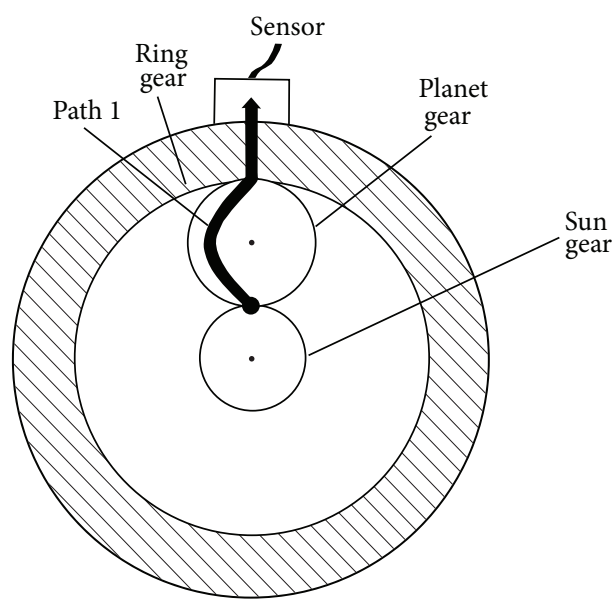

(a)

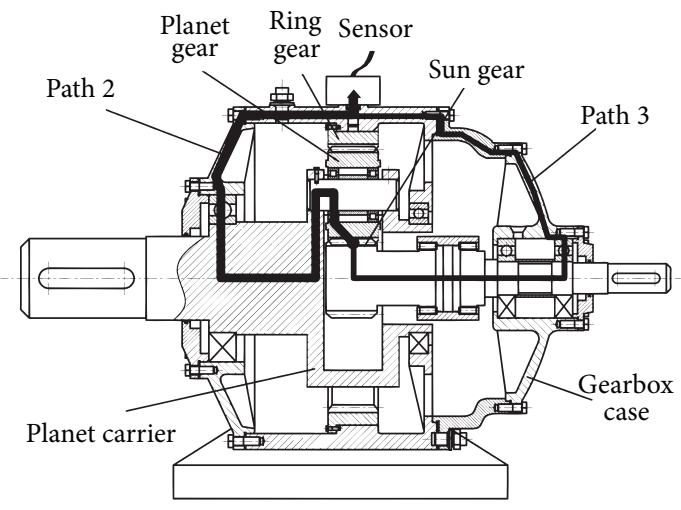

(b)

Figure 3: Propagation paths of signal. (a) Path 1; (b) path 2 and path 3.

and finally reaches the signal measurement point. There are more components in path 2 and path 3 , which causes serious signal attenuation in comparison with path 1 . Therefore, this paper only considers the signal transmitted through path 1 .

Assume that the planet gear train is simplified as a fixedshaft gear train, and a fault exists on a certain gear tooth. Due to the movement of the sensor, the relative distance between the fault area and the sensor continuously changes, given that at time $t$ the distance between the sensor and the meshing point is the closest. With the rotating movement of the sensor around the center of planetary gear train, the sensor gradually leaves from the meshing pint, and the amplitude of the vibration collected by the sensor becomes smaller. When the sensor moves to the farthest distance, the strength of vibration is the weakest one. Further movement of the sensor leads to smaller distance, and the vibration collected becomes stronger. In fact, during the spinning process of the sensor, the frequency of collected signal may have a small amount of variation. Once the sensor revolves one period around the gear train and returns to the position that has the closest distance with the meshing point, both the amplitude and the frequency of the collected signal have one period of change. Thus, the impact of sensor revolving around center of gear train can be described as amplitude modulating and frequency modulating processes.

4.3. Vibration Signal Model of Planetary Gear Train. On one hand, (17) describes the vibration signal model of the fixedshaft gear train that is transformed from the planetary gear train. On the other hand, the impact of sensor spinning is to cause amplitude modulating and frequency modulating phenomena in vibration signal. For simplification purpose, only the modulating phenomena of the fundamental frequency are considered, and the vibration signal $x(t)$ collected by sensor can be described as

$$
x(t)=A_{H 0}\left[1+A_{H 1} \cos \left(2 \pi f_{H} t+\theta_{H}\right)\right] x_{o}(t) .
$$

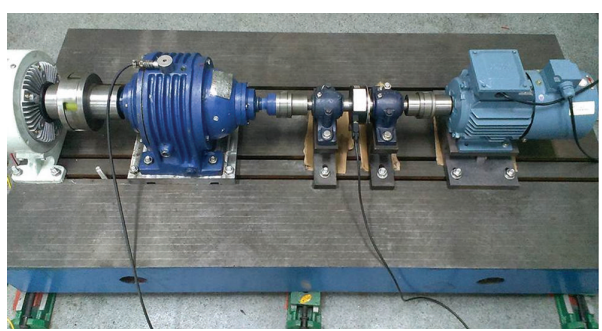

FIGURE 4: Test rig of a single-stage planetary gearbox.

Here, $x_{0}(t)$ is the original vibration signal, and its expression is shown by (17); $f_{H}$ is the relative rotating frequency of sensor, and its value is equal to the rotating frequency of planet carrier; $A_{H 0}$ is the signal attenuation coefficient; $A_{H 1}$ is the amplitude modulating strength.

Substitute (17) into (18), and the following result can be obtained:

$$
x(t)=\sum_{k=-\infty}^{+\infty} D_{k} \cos \left[2 \pi\left(f_{m}+k f_{c} \pm f_{H}\right) t+\beta_{k}\right]
$$

where $D_{k}$ and $\beta_{k}(k \in Z)$ are two constants.

Accordingly, the impact of sensor spinning on the collected vibration signal can be described as the emergence of spectral lines at the positions of $\pm f_{H}$ around sidebands $f_{m} \pm k f_{c}(k \in Z)$.

If we consider the harmonics of the meshing frequency $f_{m}$, the modulating effect, and the harmonics of $f_{H}$, the positions of sidebands are $n f_{m}+k f_{c}+l f_{H}\left(n \in N^{*}, l \in Z\right)$. Here, $f_{c}$ is the characteristic frequency of the corresponding gear fault.

It should be noted that after a period of gearbox running gears suffer certain degree of wear. In addition, errors may also be introduced during manufacturing and assembly processes. All these may cause some variations in the theoretical signal model. 


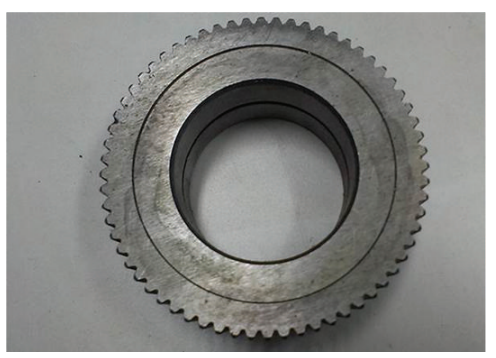

(a)

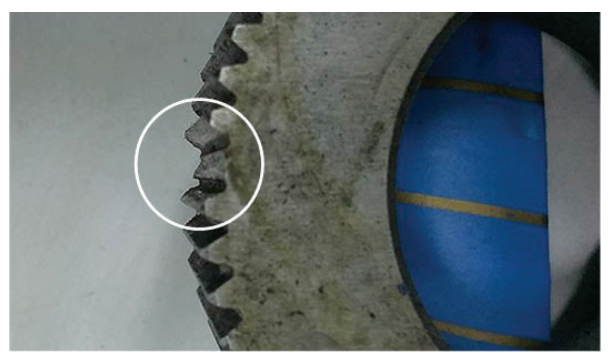

(b)

FIGURE 5: The normal planet gear and the artificially seeded faulty gear. (a) Normal planet gear; (b) artificially seeded faulty gear.

TABLE 1: Parameters of the planetary gearbox.

\begin{tabular}{lccc}
\hline $\begin{array}{l}\text { The number } \\
\text { of teeth in the } \\
\text { sun gear }\end{array}$ & $\begin{array}{c}\text { The number of } \\
\text { teeth in the } \\
\text { planet gear }\end{array}$ & $\begin{array}{r}\text { The number of } \\
\text { teeth in the ring } \\
\text { gear }\end{array}$ & $\begin{array}{c}\text { The number of } \\
\text { planet gears }\end{array}$ \\
\hline 13 & 64 & 146 & 3 \\
\hline
\end{tabular}

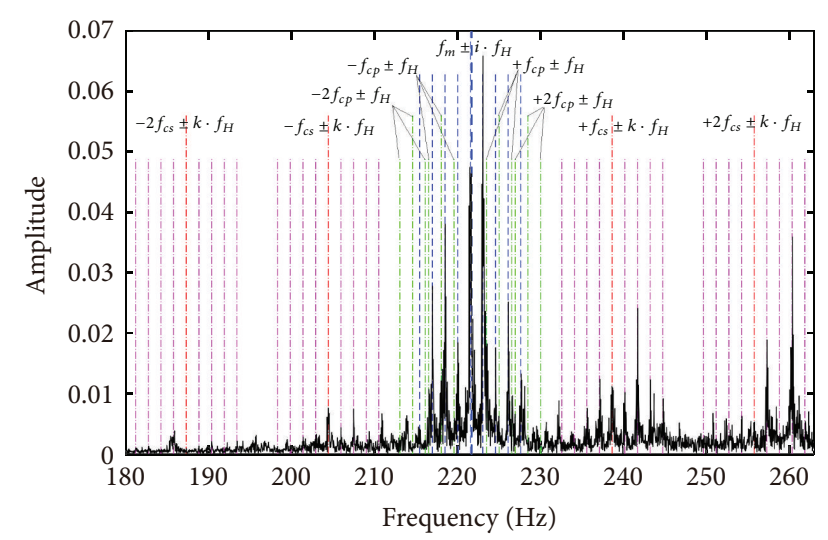

FIGURE 6: Frequency spectrum of vibration signal collected from the planetary gearbox.

\section{Case Study}

5.1. Experimental Setup. In this paper, an experiment is conducted on a single-stage planetary gearbox. The model type of gearbox is NGW11, and the gearbox transmission ratio is 12.23. Other parameters are provided in Table 1. The test rig includes a single-stage planetary gearbox, a drive motor, a magnetic powder brake, and a control and data acquisition system, which is shown in Figure 4. In the experiment, the IMI $603 \mathrm{C} 1$ sensor is mounted on the top of gearbox. The NI PCI-4472B data acquisition card is used for data collection, where the sampling frequency is $80 \mathrm{kHz}$, and the sampling time is 30 seconds. Figure 5 shows the normal planet gear and the faulty one, where the fault is artificially seeded.

5.2. Data Analysis. After a certain time of gearbox running, a piece of vibration signal was collected, and Figure 6 shows the spectrum of the signal. From Figure 6, the meshing frequency of $221.694 \mathrm{~Hz}$ can be observed, which is represented by the highest blue dashed line in the graph. The remaining blue
TABLE 2: Time-domain statistic metrics of the normal and faulty signals.

\begin{tabular}{lcc}
\hline Metric & Normal signal & Faulty signal \\
\hline Root Mean Square (RMS) & 0.5729 & 0.8820 \\
Peak Value & 3.1431 & 14.3611 \\
Crest Factor & 5.4861 & 16.2823 \\
Kurtosis & 3.4298 & 17.6152 \\
\hline
\end{tabular}

dashed lines correspond to the combination of modulating phenomena caused by the planet carrier rotation and the ring gear error. The green dash-dot lines represent the planet gear characteristic frequency and the sidebands of modulation phenomena caused by the planet carrier rotation. The red and purple dash-dot lines correspond to the sun gear characteristic frequency and the sidebands of modulation phenomena caused by the planet carrier rotation. Therefore, it can be drawn from Figure 6 that the actual sideband distribution in the signal spectrum of planetary gearbox is consistent with the vibration signal model presented in Section 4.3.

In order to validate the proposed vibration model for fault diagnosis, two pieces of vibration signals collected from the normal gearbox and the one with planet gear failure were used for comparison. Table 2 shows some time-domain statistic metrics calculated from these signals. Figure 7 shows the time-domain waveforms of the gearbox under normal condition and planet gear failure. Figure 8 shows the spectra of two signals around the first-order meshing frequency.

From Table 2, it can be seen that all the statistic metrics of the faulty vibration signal are higher than the ones of the normal signal. The increase of RMS indicates that the existence of fault causes stronger vibration. The Peak Value, Crest Factor, and Kurtosis of fault signal are several times larger than the ones calculated from the normal signal, which means there exist obvious impulses in the time-domain signal. In fact, a comparison of Figures 7 (a) and 7(b) also proves the existence of impulses in gear failure condition.

In Figure 8, the dashed lines represent the meshing frequency (the position of the highest dashed line) and the sidebands of the modulating phenomena caused by planet carrier rotation and ring gear error (the positions of remaining dashed lines). The dash-dot lines correspond to the characteristic frequency of the planet gear (the position with the highest dash-dot line) and the sidebands of the 


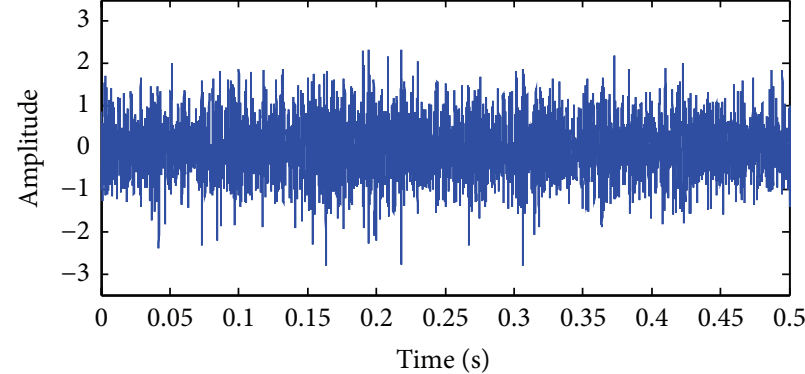

(a)

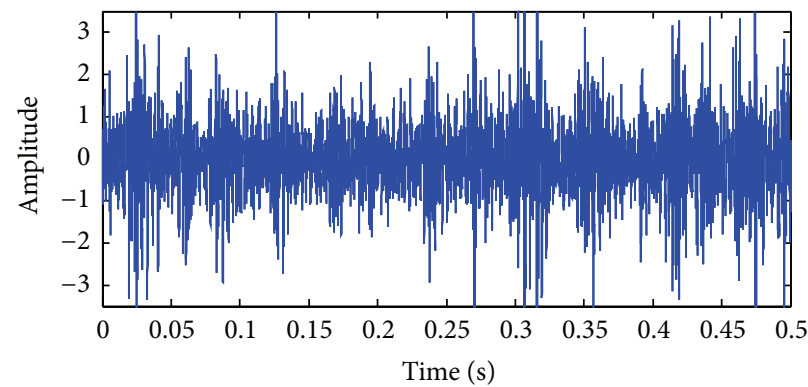

(b)

Figure 7: Time-domain signal. (a) Normal gear; (b) faulty gear.

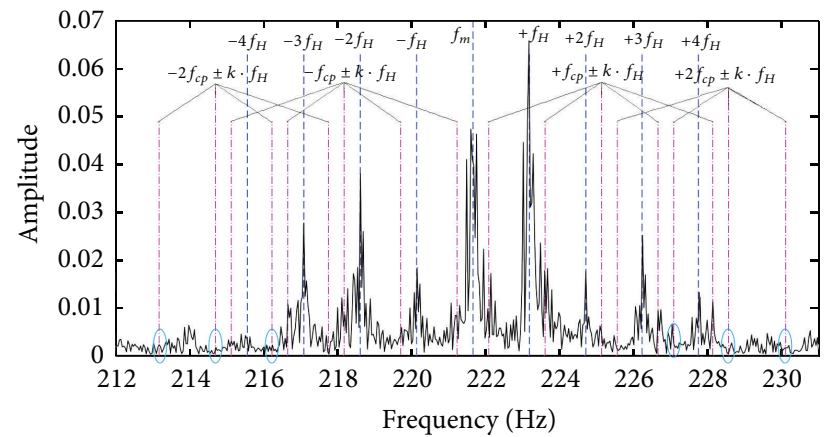

(a)

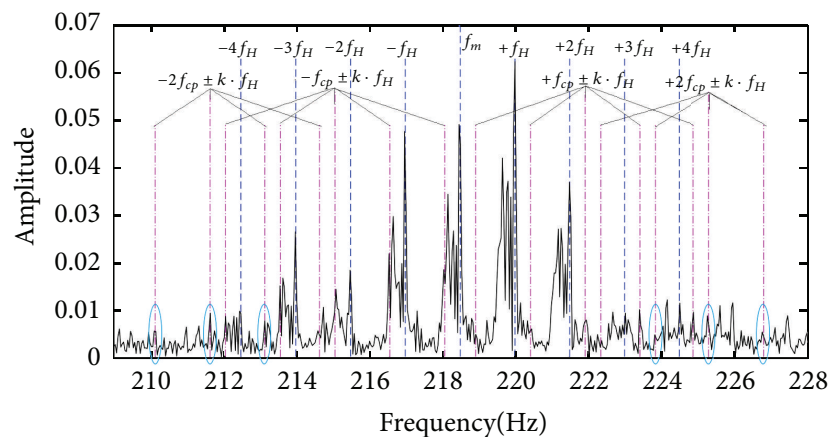

(b)

FIGURE 8: Frequency spectrum. (a) Normal gear; (b) faulty gear.

modulating phenomena caused by planet carrier rotation (the positions of remaining dash-dot lines). Through comparison between Figures 8(a) and 8(b), it can be observed that the amplitudes of the planet gear characteristic frequencies and their corresponding sidebands increase dramatically after planet gear failure. Specifically, obvious spectral lines can be found at the second harmonic of the characteristic frequency of planet gear with distributed fault (i.e., the characteristic frequency of planet gear with two-sided gear tooth fault).

Based on the aforementioned analysis results obtained from the time-domain statistic metrics, the time-domain waveform, and the emergence of sidebands, it can be concluded that there exists planet gear failure in the gearbox.

\section{Conclusions}

Planetary gearboxes have been widely used in transmission systems of helicopters, wind turbines, and engineering machinery. However, due to its complicated structure, traditional condition monitoring methods for fixed-shaft gearing system are not suitable for planetary gearbox. It is necessary to develop new methods for planetary gearbox condition and fault diagnosis.

The purpose of this research is to investigate vibration signal model of planetary gear train, which helps us to understand fault phenomena of planetary gearbox and develop reliable fault diagnosis methods. Assume that the whole gearbox reversely rotates around center of sun gear at a speed of planet gear rotation. Then, planetary gearbox fault diagnosis can be divided into two tasks: the fixed-shaft gearbox fault diagnosis and the impact of sensor spinning on vibration signal. Based on the assumptions, the vibration signal model of faulty planetary gearbox is established, and the proposed model provides us with a way to understand different fault phenomena of planetary gearbox. A test rig of planetary gearbox was built, and the vibration data collected from the test rig validated the proposed model. Further, the proposed vibration signal model successfully identified planet gear fault in the gearbox, which may be useful in developing planetary gearbox fault diagnosis methods using the information extracted from vibration signal spectrum.

\section{Conflict of Interests}

The authors declare that there is no conflict of interests regarding the publication of this paper.

\section{Acknowledgments}

This research was partially supported by the National Natural Science Foundation of China (Grant nos. 51275554 and 51405316) and the 2012 Open Project of State Key Laboratory of Mechanical Transmission, Chongqing University (Grant no. SKLMT-KFKT-201205). The authors also would like to thank Mr. Lu Yang for his help in the test rig setup in this research.

\section{References}

[1] T. M. Ericson and R. G. Parker, "Experimental measurement of the effects of torque on the dynamic behavior and system 
parameters of planetary gears," Mechanism and Machine Theory, vol. 74, pp. 370-389, 2014.

[2] W. Bartelmus, F. Chaari, R. Zimroz, and M. Haddar, "Modelling of gearbox dynamics under time-varying nonstationary load for distributed fault detection and diagnosis," European Journal of Mechanics-A/Solids, vol. 29, no. 4, pp. 637-646, 2010.

[3] X. Gu and P. Velex, "A dynamic model to study the influence of planet position errors in planetary gears," Journal of Sound and Vibration, vol. 331, no. 20, pp. 4554-4574, 2012.

[4] P. D. McFadden, "A technique for calculating the time domain averages of the vibration of the individual planet gears and the sun gear in an epicyclic gearbox," Journal of Sound and Vibration, vol. 144, no. 1, pp. 163-172, 1991.

[5] B. D. Forrester, "Method for the separation of epicyclic planet gear vibration signatures," United States Patent, US6298725 B1, 2001.

[6] M. E. Orchard and G. J. Vachtsevanos, "A particle-filtering approach for on-line fault diagnosis and failure prognosis," Transactions of the Institute of Measurement and Control, vol. 31, no. 3-4, pp. 221-246, 2009.

[7] T. Barszcz and R. B. Randall, "Application of spectral kurtosis for detection of a tooth crack in the planetary gear of a wind turbine," Mechanical Systems and Signal Processing, vol. 23, no. 4, pp. 1352-1365, 2009.

[8] Y. Lei, D. Kong, J. Lin, and M. J. Zuo, "Fault detection of planetary gearboxes using new diagnostic parameters," Measurement Science and Technology, vol. 23, no. 5, Article ID 055605, 2012.

[9] Z. P. Feng and M. J. Zuo, "Vibration signal models for fault diagnosis of planetary gearboxes," Journal of Sound and Vibration, vol. 331, pp. 4919-4939, 2012.

[10] Y. Lei, J. Lin, M. J. Zuo, and Z. He, "Condition monitoring and fault diagnosis of planetary gearboxes: a review," Measurement: Journal of the International Measurement Confederation, vol. 48, no. 1, pp. 292-305, 2014.

[11] D. Wang, Q. Miao, and R. Kang, "Robust health evaluation of gearbox subject to tooth failure with wavelet decomposition," Journal of Sound and Vibration, vol. 324, no. 3-5, pp. 1141-1157, 2009.

[12] C. Li, M. Liang, and T. Wang, "Criterion fusion for spectral segmentation and its application to optimal demodulation of bearing vibration signals," Mechanical Systems and Signal Processing, vol. 64-65, pp. 132-148, 2015.

[13] D. Wang, P. W. Tse, W. Guo, and Q. Miao, "Support vector data description for fusion of multiple health indicators for enhancing gearbox fault diagnosis and prognosis," Measurement Science and Technology, vol. 22, no. 2, Article ID 025102, 2011.

[14] D. Wang, P. W. Tse, and K. L. Tsui, "An enhanced Kurtogram method for fault diagnosis of rolling element bearings," Mechanical Systems and Signal Processing, vol. 35, no. 1-2, pp. 176-199, 2013. 

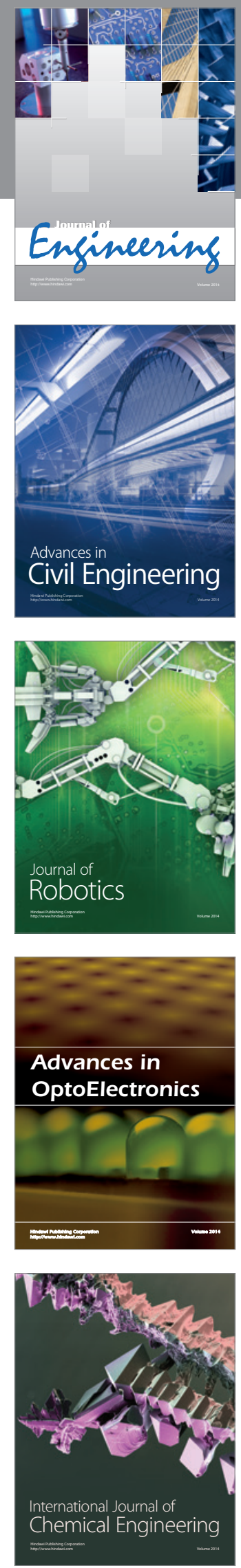

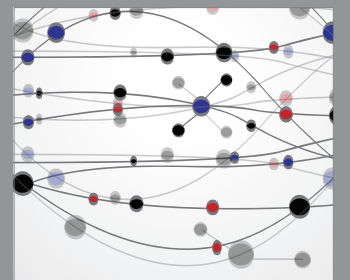

The Scientific World Journal
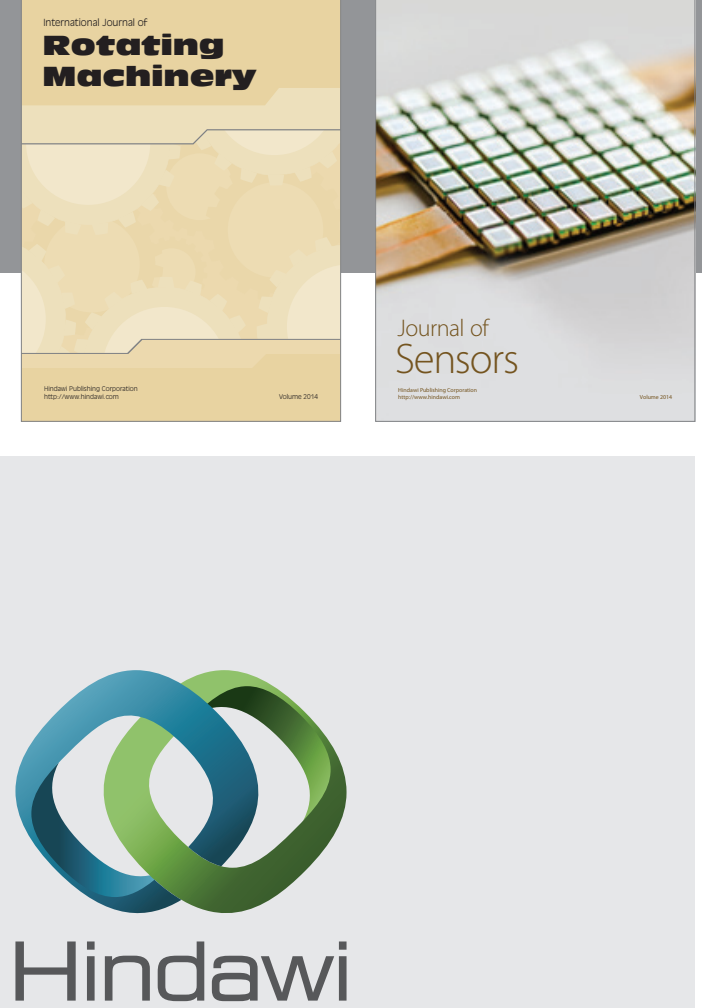

Submit your manuscripts at http://www.hindawi.com
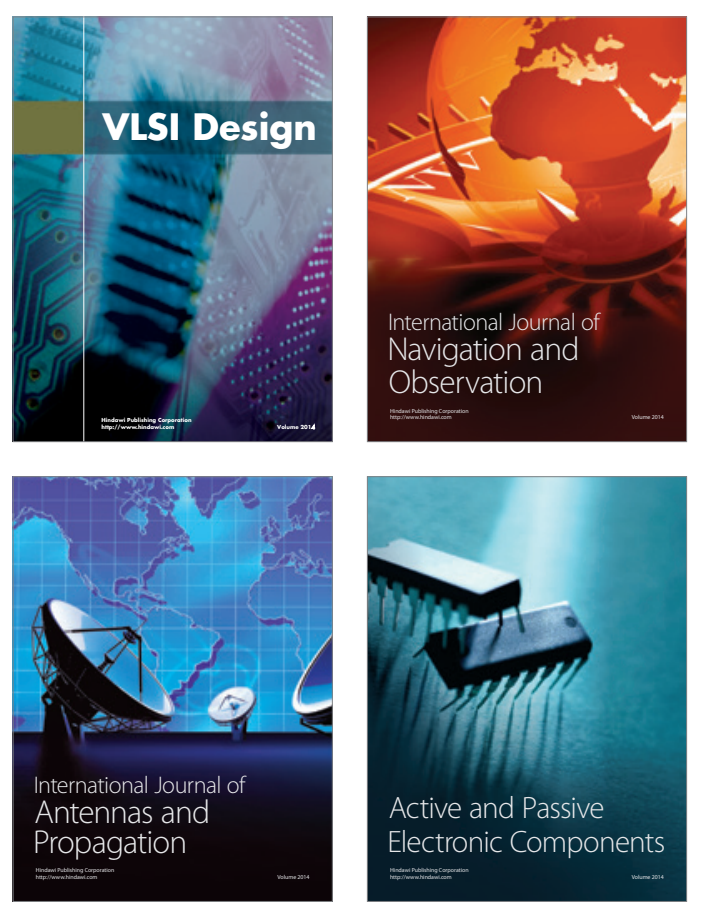
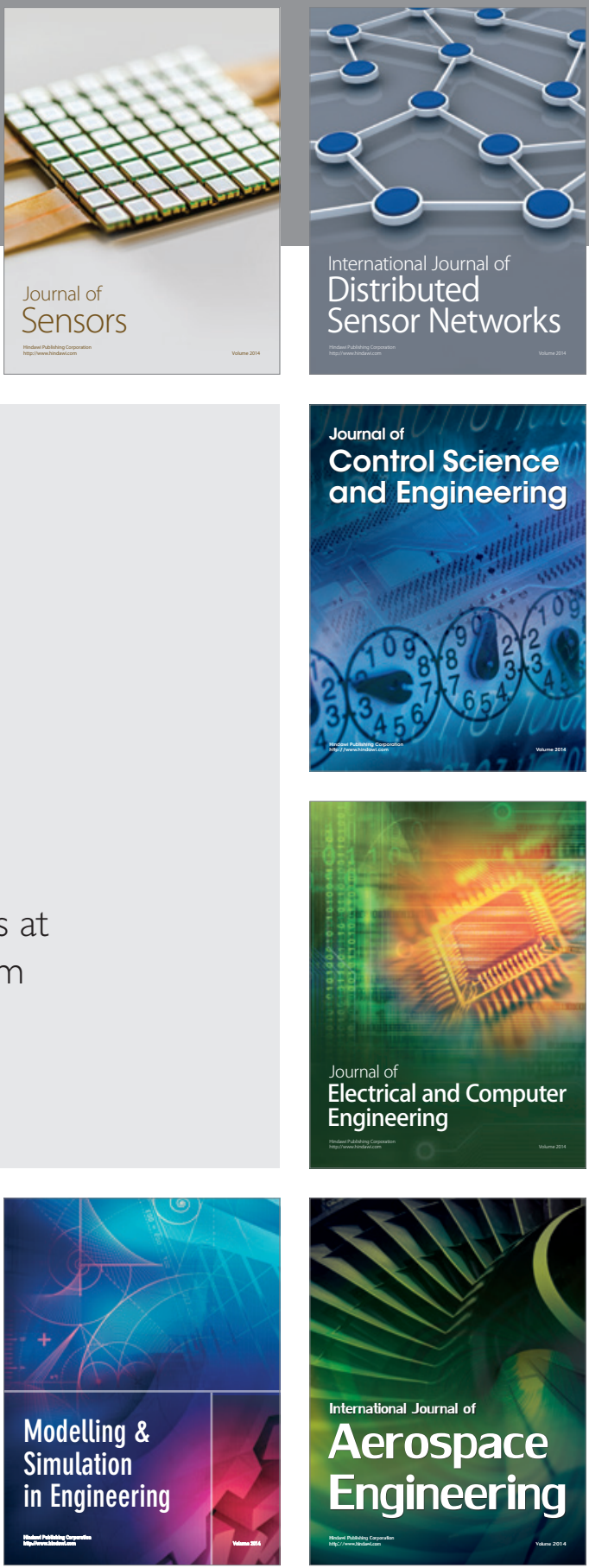

Journal of

Control Science

and Engineering
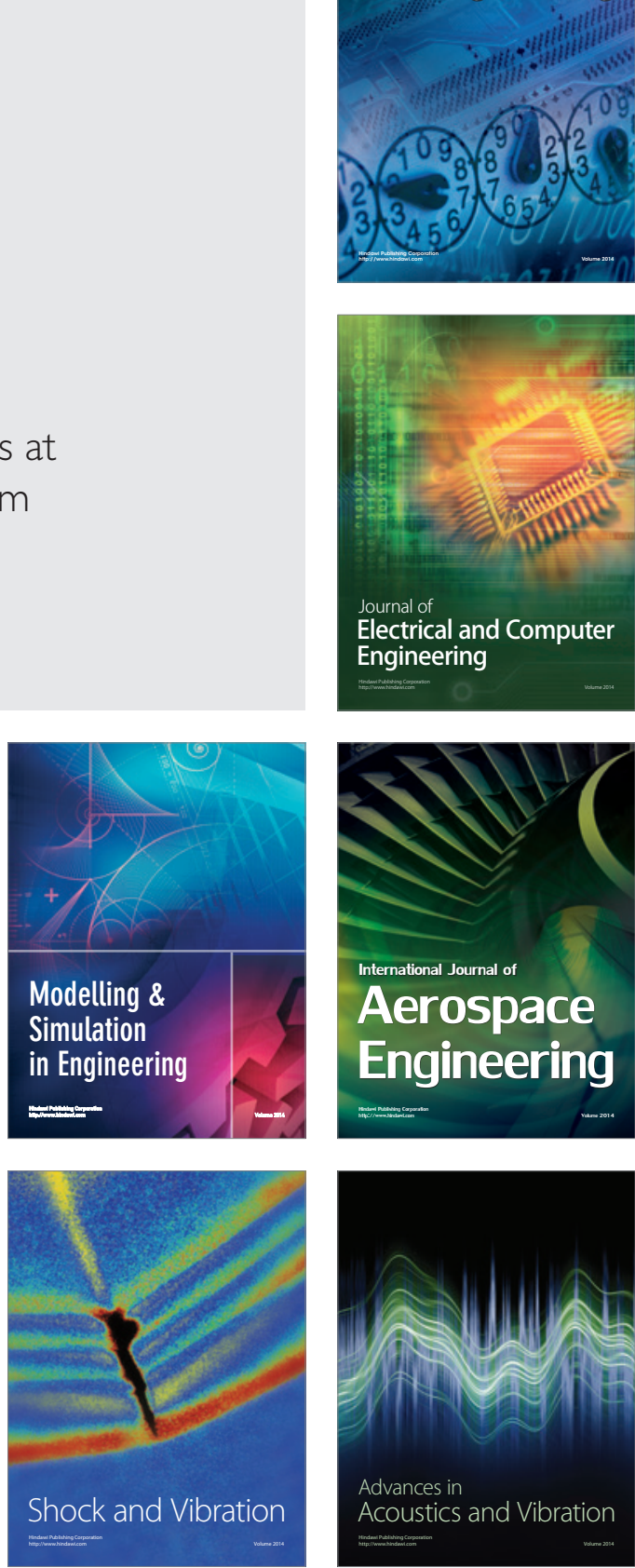